\title{
Reaction Time and Musical Expectancy: Priming of Chords
}

\author{
Jamshed Jay Bharucha and Keiko Stoeckig \\ Dartmouth College
}

\begin{abstract}
The cognitive processes underlying musical expectation were explored by measuring reaction time in a priming paradigm. Subjects made a speeded true/false decision about a target chord following a prime chord to which it was either closely or distantly related harmonically. Using a major/minor decision task in Experiment 1, we found that major targets were identified faster, and with fewer errors, when they were related than when unrelated. An apparent absence (and possible reversal) of this effect for minor targets can be attributed to the prime's biasing effect on the target's stability. In Experiments 2 and 3 we tested this hypothesis by employing an in-tune/out-of-tune decision for major and minor targets separately. Both major and minor in-tune targets were identified faster when related than when unrelated. We outline a spreading activation model which consists of a network of harmonic relations. Priming results from the indirect activation of chord nodes linked through the network.
\end{abstract}

Vital to our musical experience is the building up of expectations. In Western music, expectations are particularly pronounced in the use of harmony, the combining of tones to form chords. The most pervasive forms of Western music, popular or otherwise, employ a small set of chords in highly constrained ways. These regularities are internalized in the form of representations that influence subsequent processing (Bharucha \& Krumhansl, 1983; Krumhansl \& Kessler, 1982) and, as in other domains of cognition, define what is coherent and what is anomalous (Bharucha, Olney, \& Schnurr, 1985, Bharucha \& Pryor, in press). A musical context induces expectations by activating these representations. In this article, we demonstrate the perceptual processing of expected events relative to unexpected events.

Theoretical accounts of harmony draw heavily upon intuitions of expectation. Schenker observed that one of the qualities of the dominant chord is "to indicate that the tonic is yet to come" $(1906 / 1954$, p. 219), and that "every linear progression [in music] is comparable to a pointing of the finger-its direction and goal are clearly indicated to the ear" $(1935 / 1979$, p. 5). At the melodic level, some tones have an "upward leading" or "downward leading" quality (Schoenberg, 1954/1969, p. 23; see Bharucha, 1984). These perceived dynamic properties are not restricted to Western music. Upward- and downward-leading tones are employed with dramatic effect by performers of Indian music; the longer the resolution is delayed without diminishing the expectancy, the greater the effect of the eventual resolution. Meyer (1956), whose theory of emotion and meaning in music centers on expectation, argues that "music arouses expectations, some conscious and others unconscious, which may or may not be directly and immediately satisfied" (p. 25).

The authors thank Jack Baird, Sharona Ben-Tov, Robert Crowder, Diana Deutsch, Mari Riess Jones, Diana Raffman, Bruno Repp, and George Wolford for helpful comments.

Correspondence concerning this article should be addressed to Jamshed Jay Bharucha, Department of Psychology, Dartmouth College, Hanover, New Hampshire 03755.
Jones $(1981,1982)$ has advanced a formal theory of expectancy to capture the dynamism implied by mentalistic accounts. In her theory, a context induces a constellation of vectors, determined by previously internalized regularities, which point to anticipated events. The vectors specify ideal prototypes from which the actual events may or may not deviate. The degree and nature of this deviation determines how events are attended to and evaluated.

A convergence of data from memory confusions and relatedness judgments implicates schematic representations involved in the perception of harmony, that is, representations that embody typical harmonic relations (Bharucha \& Krumhansl, 1983; Krumhansl, Bharucha, \& Castellano, 1982). These studies indicate that the more closely related a chord is to the prior harmonic context, the greater its perceived stability and the greater the likelihood of its being confused with a chord from the context. ${ }^{1}$ They also show evidence of temporal asymmetries between two chords if one is more stable than the other: The two chords are judged more closely related and are more easily confused with each other if the more stable one follows the less stable one than vice versa. In the absence of a harmonic context, major chords are more stable than minor chords and exhibit a major-minor temporal asymmetry.

In Figure 1 we outline a spreading activation model of the representation and processing of harmony, which accounts for available data and motivates the present experiments. Nodes represent chords (limited here to major and minor chords) and

\footnotetext{
' A key is established by the use of tones from a familiar musical scale or chords based on those tones (the daughter chords of a key). A key thus has several daughter chords (including both major and minor chords), and a chord may have several parent keys. Keys that share daughter chords are closely related, and chords that share parent keys are closely related.

A chord is stable to the extent that it sounds final, or not in need of resolution. Stability contributes to, perhaps even underlies, consonance. Piston (1978), for example, characterizes a consonant sound as "stable and complete" and a dissonant sound as "restless" and "in need of resolution" (p. 6).
} 


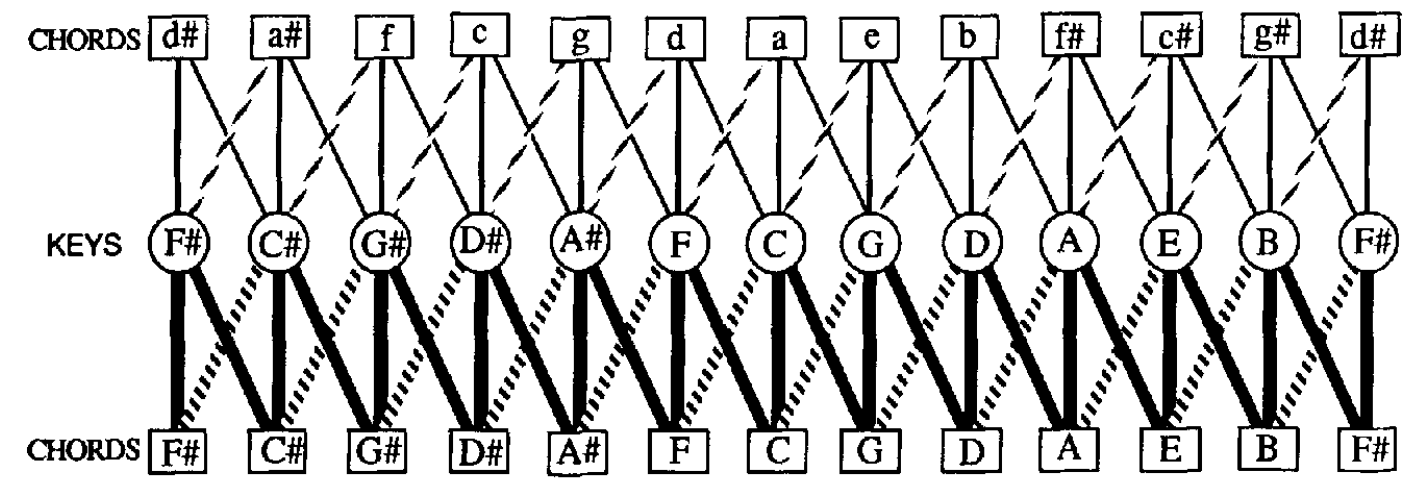

Figure 1. A spreading activation network linking chords and their parent keys. (Link strengths determine the amount of activation transmitted and are indicated by the thickness and solidity of lines connecting chord and key nodes. Major and minor chords are indicated by upper and lower case letters, respectively, and are located on different sides of the key nodes for convenience only. When a chord is heard, activation spreads through the network in accord with the link strengths, decaying over time.)

keys (limited here to major keys). Each chord node is linked to its parent key nodes (see Footnote 1). (Major and minor chord nodes are labeled with uppercase and lowercase letters, respectively, and are shown on different sides of the key nodes for convenience only.) The structure in Figure 1 wraps around so that the left and right edges are identical. If minor keys are incorporated, the network architecture would embody the constraints described by Krumhansl and Kessler (1982). A link's strength (indicated in Figure 1 by the thickness and solidity of a link) corresponds to the strength of a chord's harmonic function in (or the degree to which the chord establishes) a parent key. The stronger the link beween nodes, the greater the activation transmitted. When a chord is sounded (either harmonically or melodically), activation spreads from the corresponding chord node to its parent key nodes, then to their daughter chord nodes (including reverberatory activation to the original chord node), and so on, through the network, decaying over time.

The more highly activated a chord node is, the more stable the chord is perceived to be, and, in a memory task, the greater the likelihood of its being confused with a chord in the prior context. The relatedness of two chords is based on the activation of the last chord, resulting in the major-minor temporal asymmetry because of the greater reverberatory activation of the major chord. Harmonic expectations result from the indirect activation of chord nodes. A chord activates (primes) the nodes of related chords more than the nodes of unrelated chords; thus the former are more expected than the latter.

In the present set of experiments, we tested the hypothesis that the prior activation of a node speeds up the processing of that chord. We presented subjects with a context chord (the prime) followed by a chord (the target) about which subjects were instructed to make a speeded true/false decision. We adopted a major/minor decision in the first experiment and an in-tune/out-of-tune decision in the second and third experiments. The prime and target chords were either closely related (henceforth referred to as related) or distantly related (henceforth referred to as unrelated). We predicted faster decision times for related than for unrelated targets.

Three qualifications need mentioning when discussing musi- cal expectancy. First, the listener's expectations need not be explicit or, as Meyer (1956) points out, need not be conscious. A reaction time paradigm should be able to detect any facilitation, whether conscious or not. Second, the expectancies driven by ingrained cognitive structures are not to be confused with the expectancies that arise from familiarity with a particular piece (Dowling \& Harwood, 1985; Rosner \& Meyer, 1982). An argument that has often been advanced as a reductio ad absurdum against expectationist accounts of music is that once we are familiar with a piece of music, we know exactly what to expect. Yet a "deceptive cadence" sounds deceptive even when we know it's coming. Third, a theory based on expectations need not be prescriptive, because a composer is free to deviate from these expectations to achieve the desired effect. Meyer (1956) and Mandler (1984) argue that departures from the expected are important determinants of the aesthetic value of a piece.

\section{Experiment 1}

The first experiment involved a major/minor decision about the target chord. The prime was either major or minor; the target was either major or minor; and the prime and target were either related or unrelated. In the related condition, the prime and target shared parent keys. In the unrelated condition, they shared no parent keys, and their parent keys shared no daughter chords. For example (see Table 1 and Figure 1), when the prime was $\mathrm{C}$ major, the related major target was $\mathrm{F}$ major, the unrelated major target was F\# major, the related minor target was A minor, and the unrelated minor target was $\mathrm{D \#} \mathrm{minor.} \mathrm{When} \mathrm{the}$ prime was $\mathrm{A}$ minor, the related major target was $\mathrm{C}$ major, the unrelated major target was $\mathrm{F} \#$ major, the related minor target was $\mathrm{D}$ minor, and the unrelated minor target was $\mathrm{D \#}$ minor.

Subjects were first given a training session in which they made major/minor judgments with feedback, in the absence of a prime, and with no time pressure. Subjects who reached a criterion level of performance proceeded to the main experiment. We predicted that target chords would be identified more quickly when related to the prime than when unrelated. 
Table 1

Examples of the Prime-Target Pairs in

Experiments 1, 2, and 3

\begin{tabular}{cll}
\hline & \multicolumn{2}{c}{ Target } \\
\cline { 2 - 3 } Prime & \multicolumn{1}{c}{ Related } & Unrelated \\
\hline Experiment 1 & F major & F\# major \\
C major & A minor & D\# minor \\
& C major & F\# major \\
A minor & D minor & D\# minor \\
Experiment 2 & G major & F\# major \\
C major & $\sim$ G major & $\sim$ F* major \\
Experiment 3 & A minor & G\# minor \\
C major & $\sim$ A minor & $\sim$ G\# minor \\
&
\end{tabular}

Note. $\sim$ indicates out-of-tune chord.

\section{Method}

Subjects. Thirteen members of the Dartmouth community participated as subjects. Nine were introductory psychology students who participated for credit. Subjects were required to be able to distinguish between major and minor chords. Musical training was measured by the total number of instrument-years of instruction. All subjects but 1 had at least 5 years of training, with an average of 9.4 years and a median of 9 years. Six subjects reported having taken at least one music theory course at the college level. All subjects reported having normal hearing, and none reported having absolute pitch.

Apparatus. Chords were synthesized by an Apple Macintosh computer and presented to subjects through a Sansui A-707 amplifier and Sennheiser HD-410 headphones.

Stimuli. Chords were synthesized according to an equal tempered tuning (in which one semitone is a frequency factor of $2^{1 / 12}$ ) ranging from $440 \mathrm{~Hz}$ to $1244 \mathrm{~Hz}$. The waveform of each component tone contained the first four harmonics with equal amplitudes. All chords were in root position (i.e., the root tone of the chord was the lowest in pitch). Major chords consisted of the root tone plus the fourth and seventh semitones above the root. Minor chords consisted of the root tone plus the third and seventh semitones above the root.

Each of the 12 major and 12 minor chords (i.e., using each of the 12 chromatic tones as roots) occurred as a prime on four different trials, paired once with each of four targets: related major, unrelated major, related minor, and unrelated minor. All 24 major and minor chords appeared as primes before any could reappear as a prime. Each subject received the 96 trials in a different random order.

The prime-target pairs, specified in terms of distance between their roots, were as follows. When the prime was major, the related major target was five semitones above, the unrelated major target was six semitones above, the related minor target was nine semitones above, and the unrelated minor target was three semitones above. When the prime was minor, the related major target was three semitones above, the unrelated major target was nine semitones above, the related minor target was five semitones above, and the unrelated minor target was six semitones above

Procedure. Subjects, run individually, were first given a training session designed to ensure that they were able to discriminate between major and minor before beginning the main experiment. On each trial of the training session, a chord was played for $2 \mathrm{~s}$, and subjects were instructed to respond "major," "minor," or "don't know" by pressing one of three designated keys on the computer keyboard. The "don't know" response caused the chord to be played again, giving subjects a second chance to respond. Subjects were judged to be reliable discriminators when they obtained an accuracy of $90 \%$ in a block of 20 chords with no "don't know" responses. Subjects trained until they either reached criterion or withdrew from the experiment. Subjects who reached criterion continued immediately to the main experiment.

In the main experiment, subjects initiated each trial by pressing the space bar. The prime chord then played for $3 \mathrm{~s}$, immediately followed by the target chord for $2 \mathrm{~s}$. Subjects were instructed to decide, "as quickly as possible while being as accurate as possible," whether the target chord was major or minor, and to respond by pressing the appropriately marked key. Subjects were given five practice trials at the beginning of the session, with more practice if requested. Practice trials were randomly drawn from the test set. The entire session lasted about $30 \mathrm{~min}$, after which subjects were debriefed and asked to fill out a musical background questionnaire.

\section{Results}

Mean reaction times (for correctly identifying the target as major or minor) and error rates are shown in Figure 2. Reaction times greater than $2,000 \mathrm{~ms}$ ( $3.5 \%$ of all reaction times) or less than zero $(0.4 \%$ of all reaction times) were discarded. Reaction times and error rates were each analyzed using an analysis of variance with three factors: relatedness (related vs. unrelated), target (major vs. minor), and prime (major vs. minor).

There was a main effect of relatedness, $F(1,12)=12.21, p<$ .01 , for reaction times; $F(1,12)=14.19, p<.01$, for errors; and there was an interaction between relatedness and target, $F(1$, $12)=21.98, p<.001$, for reaction times; $F(1,12)=32.88, p<$ .001 , for errors. The main effect was carried entirely by the major targets, for which responses were much faster and more accurate when related than when unrelated. For minor targets, responses were slower and less accurate when related than when unrelated, but not significantly so (by a separate analysis of variance for minor targets).

All 13 subjects showed the reaction time advantage for related over unrelated for major targets. The size of this effect ranged from $36 \mathrm{~ms}$ to $403 \mathrm{~ms}$, with a mean of $176 \mathrm{~ms}$, and was not correlated with years of musical training $(r=-.295)$.

The overall advantage for related over unrelated was slightly more pronounced for major primes than for minor primes, but only for error rates was this interaction significant, $F(1,12)=$ $14.53, p<.01$.

Relative to minor primes, major primes caused a greater separation between major and minor targets, with slower and less accurate responses to major targets, and faster and more accurate responses to minor targets, as indicated by a Prime $\times$ Target interaction-significant for reaction time, $F(1,12)=8.68$, $p<.05$, but not for errors, $F(1,12)=3.92, p<.1$.

There was a strong bias to judge targets to be major when related and minor when unrelated. The proportion of targets judged to be major was significantly greater than chance when related $(.54), t(12)=1.93, p<.05$, and significantly less than chance when unrelated (.38), $t(12)=5.76, p<.0001$, where chance was .5.

Reaction times for true identifications were computed by factoring out correct guesses from the correct responses (see Wolford, Wessel, \& Estes, 1968), and exhibited the same pattern as the correct identification times. 


\section{Major prime}
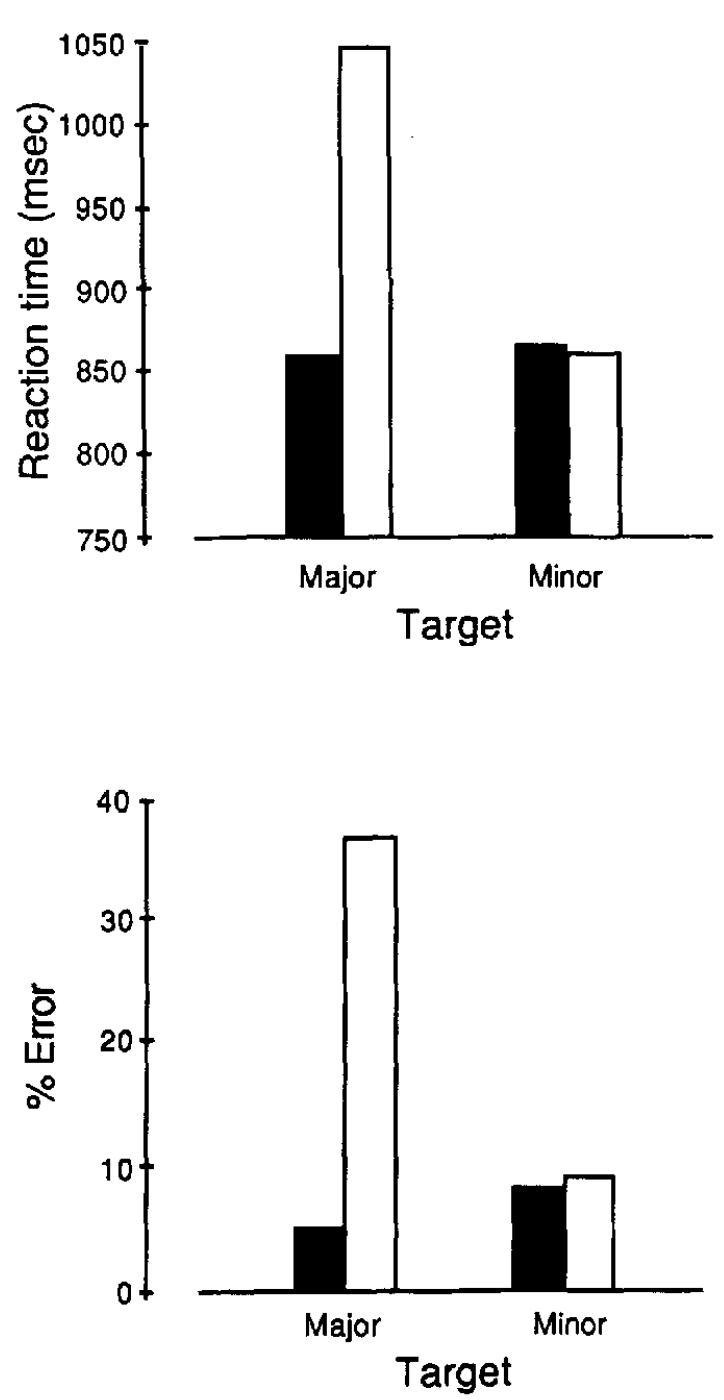

Minor prime
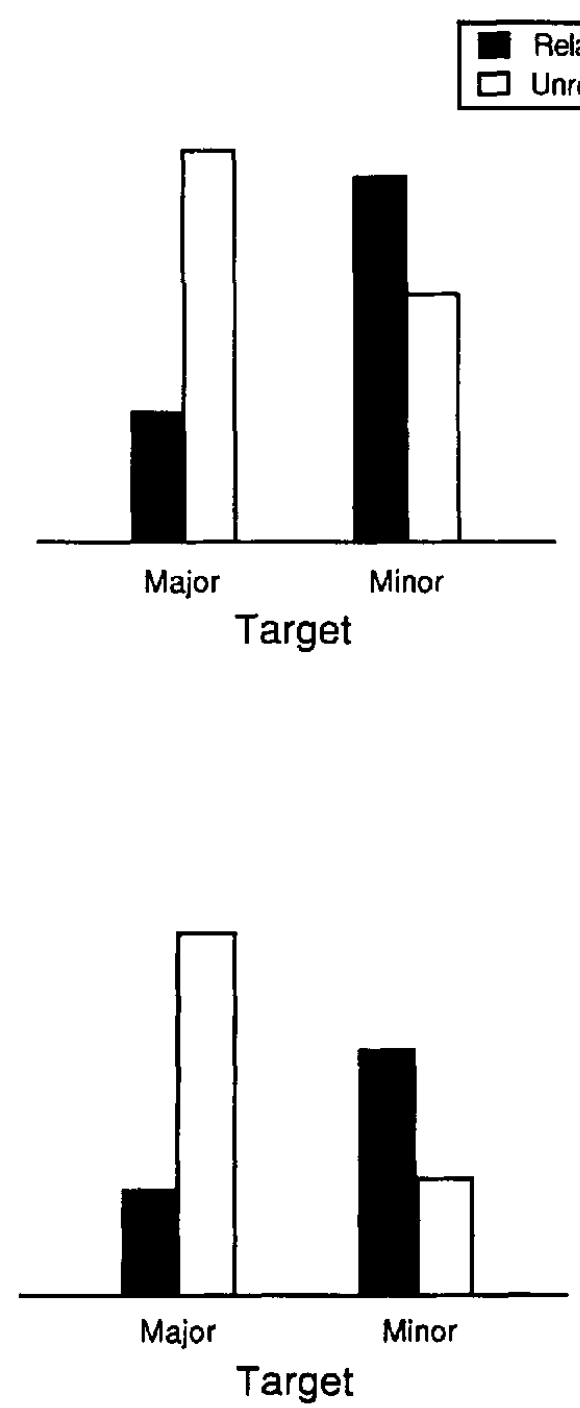

Figure 2. Subjects made a speeded major/minor decision about a target chord. For major targets, subjects were faster (top) and made fewer errors (bottom) when the prime and target were related than when unrelated. For minor targets, the effect was reversed, (though insignificant).

The following comparisons are based on a Newman-Keuls test applied separately to the related and unrelated conditions, using $p<.05$ as the criterion for significance. When related, a major prime followed by a minor target resulted in insignificantly slower reaction times and insignificantly more errors than a minor prime followed by a major target. When unrelated, a major prime followed by a minor target resulted in significantly faster reaction times and significantly fewer errors than a minor prime followed by a major target.

\section{Discussion}

Major targets were identified much faster and more accurately when they were related than when unrelated. However, minor targets were identified slightly (though insignificantly) more slowly and less accurately when related than when unrelated. We hypothesize that the overall advantage of related over unrelated targets reflects the effect of priming on sensitivity to the target, and the Relatedness $\times$ Target interaction reflects the effect of priming on the target's stability or consonance.

Related targets were more likely to be judged major, and unrelated targets were more likely to be judged minor. For related targets, this bias in favor of major quickens responses to major targets and slows down responses to minor targets. For unrelated targets, the bias in favor of minor slows down responses to major targets and quickens responses to minor targets, yielding the observed Relatedness $\times$ Target interaction.

We suggest that the prime's influence on the target's stability was responsible for this shift in bias. Major chords are perceived to be more stable than minor chords, and a harmonic context 
increases the perceived stability of closely related chords and decreases the perceived stability of distantly related chords (Bharucha \& Krumhansl, 1983; Krumhansl, Bharucha, \& Castellano, 1982; Krumhansl, Bharucha, \& Kessler, 1982). If the difference in stability between major and minor chords influenced the decision, the increased stability of a related target would bias the decision in favor of major, relative to an unrelated target. In terms of spreading activation, major target chords cause greater activation of their nodes than do minor target chords, independent of prior context, because of greater reverberatory activation from their parent keys. If the activation level of the target chord node is used to help guess if it is major or minor, prior activation (from a related prime) will bias the decision in favor of major.

This hypothesis receives support from judgments of consonance, which are higher for major chords than for minor chords (Roberts, 1983), and which, Roberts and Shaw (1984) argue, influence same-different judgments of chord type (among them major and minor).

When the prime-target pair consisted of one major and one minor chord, reaction times differed as a function of temporal order. When related, responses were faster for major targets; when unrelated, responses were faster for minor targets. These asymmetries are a direct result of the prime's biasing influence. The asymmetry observed with relatedness judgments (Bharucha \& Krumhansl, 1983; Krumhansl, Bharucha, \& Kessler, 1982) thus manifests itself in two different directions in a speeded decision task, because of the greater confidence with which a decision can be made as the pair gets more related or more unrelated.

In the next two experiments we explored the interpretation given to the Relatedness $\times$ Target interaction by testing major and minor targets in separate experiments, using a foil less consonant than each. The foil was an out-of-tune chord, and subjects made an in-tune/out-of-tune decision. If chord nodes are activated by frequency-specific channels (as in Deutsch, 1969), then chord nodes should be activated less by out-of-tune chords than by in-tune chords. This should result in a bias to respond "in-tune" to related targets and "out-of-tune" to unrelated targets, causing a Relatedness $\times$ Intonation (in-tune vs. out-oftune) interaction.

We restricted ourselves to major primes because the interaction between relatedness and prime indicated that major primes had a stronger priming effect. This is consistent with the greater strength with which major chords establish a key (Krumhansl \& Kessler, 1982; Roberts \& Shaw, 1984) and reflects the stronger links emanating from major chord nodes.

\section{Experiment 2}

This experiment involved an in-tune/out-of-tune decision for major targets. The particular chord pairs for the related condition differed from Experiment 1 in order to generalize the result to a different related chord. When the prime was $\mathrm{C}$ major, for example (see Table 1 and Figure 1), the related target was $G$ major, and the unrelated target was $F \#$ major.

Because the chords in Experiment 1 were in root position, and the component tones of a given chord were from the same octave, the prime-target pair involved a leap in pitch that is not typical of music. In order to better control pitch height, chords in this and the next experiment were sampled from each of five octaves simultaneously, and their component tones tapered off toward threshold at either end of the range. These chords come as close as possible to being pure chord functions stripped of pitch height and inversion (see Krumhansl, Bharucha, \& Kessler, 1982; Shepard, 1964).

In order to minimize the influence of the previous trial, each trial was preceded by a rapid sequence of 16 tones drawn at random from the frequency continuum- a different random sequence for each trial.

\section{Method}

Subjects. Twelve subjects from the Dartmouth community participated in the experiment, 9 of whom received partial credit for the introductory psychology course. Musical background ranged from no training to 11 years of training, with an average of 5.9 years and a median of 6 years. Three subjects reported having taken at least one music theory course. All subjects reported having normal hearing, and none reported having absolute pitch.

Apparatus. Same as in Experiment 1.

Stimuli. Primes consisted of the 12 major chords based on an equal tempered tuning fixed at an A of $440 \mathrm{~Hz}$. Targets consisted of the same 12 chords plus 12 mistuned versions created by lowering the frequency of the fifth degree by a factor of $2^{1 / 48}$ (an eighth-tone). Chords were constructed by imposing an envelope over a five-octave range (starting at $65.41 \mathrm{~Hz}$ ), tapering off to the loudness threshold at each end. The three component tones of each chord were sampled from each of these five octaves and added in proportion to their envelope amplitudes. The waveform of each of the 15 component tones contained the first four harmonics with equal amplitudes.

Each of the 12 major chords occurred as a prime, paired with each of four targets: in-tune related major, in-tune unrelated major, out-oftune related major, and out-of-tine unrelated major. Each subject received the 96 trials in a different random order.

The prime-target pairs, specified in terms of distance between their roots, were as follows. The related target was seven semitones above, and the unrelated target was six semitones above. Each target was either in-tune or out-of-tune.

The 16 tones of random pitch preceding the trial were "Shepard" tones, shaped as indicated above, and their frequencies were picked at random for each trial. Each lasted $125 \mathrm{~ms}$, with no pause between tones.

Procedure. All subjects ran through a training session consisting of three examples each of in-tune and out-of-tune chords, followed by 48 trials in which subjects had to distinguish between in-tune and out-oftune chords, without instructions to maximize speed. Subjects were allowed to proceed to the main experiment only after achieving an accuracy of $90 \%$ or better and were permitted to repeat the training session until the criterion accuracy was reached. This task proved to be much easier than the major/minor decision, and permitted us to use subjects with all levels of musical training, including those with no training at all.

In the main experiment, subjects initiated each trial by pressing the space bar. A trial began with a sequence of 16 tones of random pitch, followed by a $1-s$ pause. This random sequence was presented to mask the influence of the previous trial.

One second after the random sequence, the prime chord was presented for $3 \mathrm{~s}$, followed immediately by the target chord for $2 \mathrm{~s}$. Subjects were instructed to decide, "as quickly as possible while being as accurate as possible," whether the target chord was in-tune or out-of-tune and to respond by pressing the appropriately marked key. Subjects were told after each response whether it was correct or incorrect. Summary feedback of mean reaction time and accuracy was given after every 24 trials. 

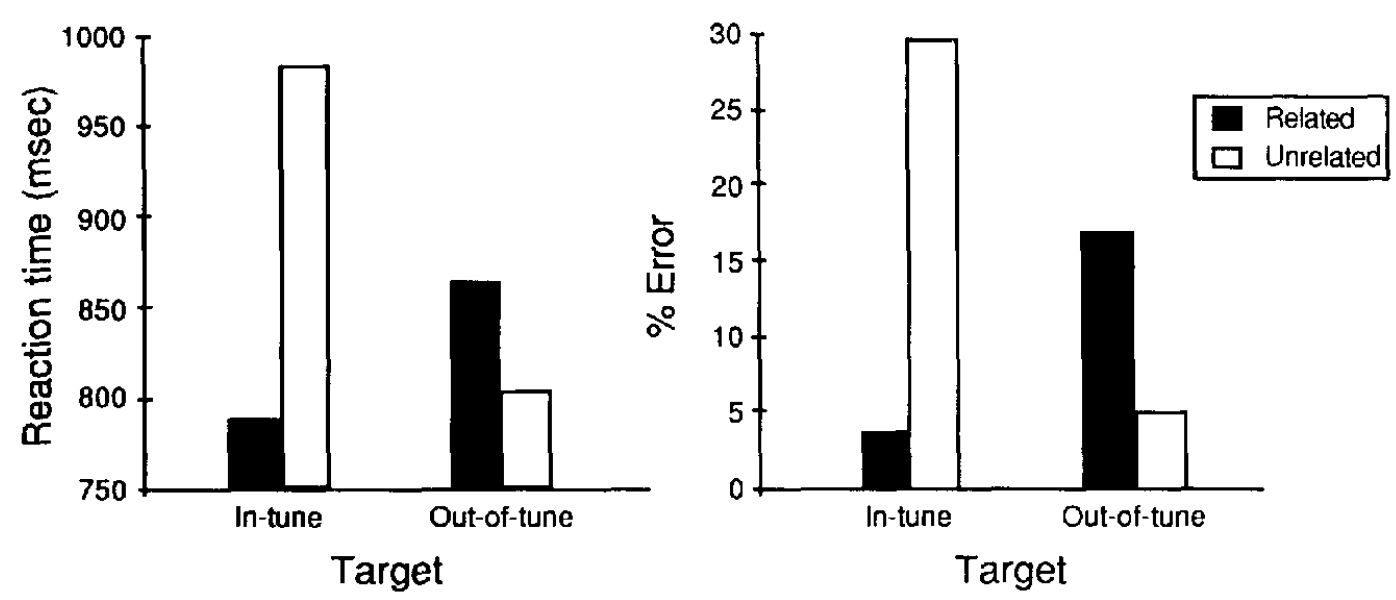

Figure 3. Subjects made a speeded in-tune/out-of-tune decision about a major target chord. For in-tune targets, subjects were faster (left) and made fewer errors (right) when the prime and target were related than when unrelated. The difference was in the reverse direction for out-of-tune targets, though not as large as for in-tune targets.

Subjects were given 12 practice trials at the beginning of the session, with more practice if requested. Practice trials were randomly drawn from the test set. The entire session lasted about $45 \mathrm{~min}$, after which subjects were debriefed and asked to fill out a musical background questionnaire.

\section{Results}

Mean reaction times (for correctly identifying the target as in-tune or out-of-tune) and error rates are shown in Figure 3. Reaction times greater than $2,000 \mathrm{~ms}(2.3 \%$ of all reaction times) or less than zero $(0.69 \%$ of all reaction times) were discarded. Reaction times and error rates were each analyzed using an analysis of variance with two factors: relatedness (related vs. unrelated) and intonation (in-tune vs. out-of-tune).

As predicted, there was a main effect of relatedness, $F(1$, $11)=6.45, p<.05$ for reaction times; $F(1,11)=14.28, p<.01$ for errors; and there was an interaction between relatedness and intonation, $F(1,11)=19.11, p<.01$, for reaction times; $F(1$, $11)=17.67, p<.01$ for errors. For in-tune targets, responses were much faster and more accurate when related than when unrelated. For out-of-tune targets, responses were slower and less accurate when related than when unrelated $F(1,11)=4.71$, $p=.05$, for reaction time; $F(1,11)=14.36, p<.01$, for errors (by a separate analysis of variance for out-of-tune targets).

There was a strong bias to judge targets to be in-tune when related and out-of-tune when unrelated. The proportion of targets judged to be in-tune was significantly greater than chance when related $(.57), t(11)=5.08, p<.001$, and significantly less than chance when unrelated $(.38), t(11)=3.47, p<.01$, where chance was 5.

Eleven out of the 12 subjects showed the reaction time advantage for related in-tune targets over unrelated in-tune targets. The size of this effect for the 11 ranged from $46 \mathrm{~ms}$ to $526 \mathrm{~ms}$. The 12th subject, with 3 years of training, showed an $81-\mathrm{ms}$ difference. Reaction time differences were not correlated with musical training $(r=.192)$.
True identification times, corrected for guessing, showed similar patterns.

\section{Discussion}

In-tune targets were identified more quickly, and out-of-tune targets more slowly, when they were related than when unrelated. The difference was much greater for the in-tune targets than for the out-of-tune targets, as indicated by overall faster reaction times for related than for unrelated targets. Furthermore, related targets were more likely to be judged in tune, and unrelated targets were more likely to be judged out of tune. This reveals an effect of priming on both sensitivity and bias; the bias enhances the advantage of related over unrelated for the more consonant target (in tune) but diminishes it for the less consonant target (out of tune) because of an influence of relatedness on consonance.

The in-tune/out-of-tune task provided a stronger demonstration of priming than did the major/minor task because subjects with less musical training were able to reach the criterion of performance in order to participate in the experiment.

\section{Experiment 3}

This experiment involved an in-tune/out-of-tune decision for minor targets, and was in all other respects identical to Experiment 2 . In the related condition, the same prime-target pair from Experiment 1 was used. The unrelated condition was changed so that the distance between the related and unrelated targets was the same as in Experiment 2. When the prime was C major, for example (see Table 1 and Figure 1), the related target was A minor, and the unrelated target was $\mathrm{G \#} \mathrm{minor.}$

\section{Method}

Subjects. Fifteen subjects from the Dartmouth community participated in the experiment, 11 of whom received partial credit for the introductory psychology course. Musical background ranged from no 

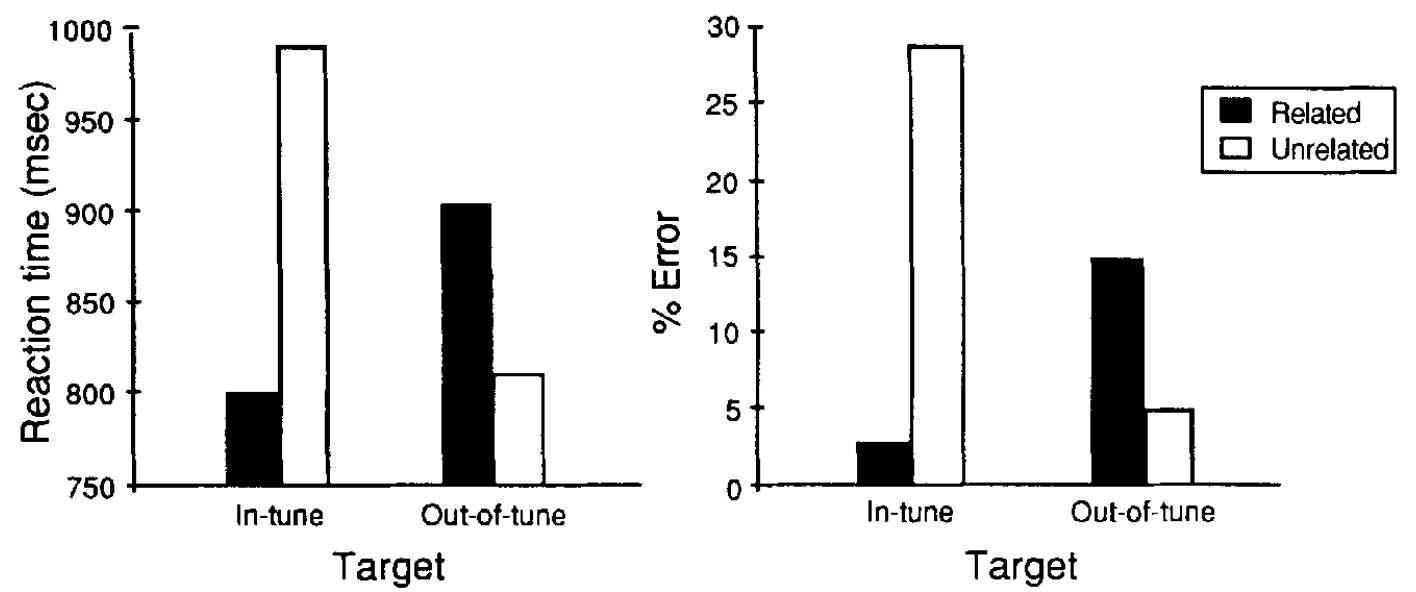

Figure 4. Subjects made a speeded in-tune/out-of-tune decision about a minor target. For in-tune targets, subjects were faster (left) and made fewer errors (right) when the prime and target were related than when unrelated. The difference was in the reverse direction for out-of-tune targets, though not as large as for intune targets.

training to 20 years of training, with an average of 7.2 years and a median of 8.5 years. Five subjects reported having taken at least one music theory course. All subjects reported having normal hearing, and none reported having absolute pitch.

Stimuli. Primes consisted of the 12 major chords based on an equal tempered tuning fixed by an $\mathrm{A}$ of $440 \mathrm{~Hz}$. Targets consisted of the 12 minor chords plus 12 mistuned versions created by lowering the frequency of the fifth by a factor of $2^{1 / 48}$ (an eighth-tone). Chords were shaped as described in Experiment 2.

Each of the 12 major chords occurred as a prime, paired with each of four targets: in-tune related minor, in-tune unrelated minor, out-oftune related minor, and out-of-tune unrelated minor. Each subject received the 96 trials in a different random order.

The prime-target pairs, specified in terms of distance between their roots, were as follows. The related target was nine semitones above, and the unrelated target was eight semitones above. Each target was either in-tune or out-of-tune.

Procedure. The procedure was the same as for Experiment 2, except that the criterion of accuracy in the training session was lowered to $75 \%$ because discriminating in-tune and out-of-tune was much more difficult for minor chords than for major chords, given the same frequency ratio of mistuning.

\section{Results}

Mean reaction times (for correctly identifying the target as in-tune or out-of-tune) and error rates are shown in Figure 4. Reaction times greater than $2,000 \mathrm{~ms}(1.3 \%$ of all reaction times) or less than zero ( $0.07 \%$ of all reaction times) were discarded. Reaction times and error rates were each analyzed using an analysis of variance with two factors: relatedness (related vs. unrelated) and intonation (in-tune vs. out-of-tune).

As predicted, there was a main effect of relatedness, $F(1$, $14)=8.28, p<.05$, for reaction times; $F(1,14)=18.26, p<$ .001 for errors; and there was an interaction between relatedness and intonation, $F(1,14)=28.80, p=.0001$, for reaction times; $F(1,14)=18.84, p<.001$, for errors. For in-tune targets, responses were much faster and more accurate when related than when unrelated. For out-of-tune targets, responses were slower and less accurate when related than when unrelated, $F(1$, 14) $=17.22, p<.001$, for reaction time; $F(1,14)=6.05, p<$ .05 , for errors (by a separate analysis of variance for out-of-tune targets).

There was a strong bias to judge targets to be in tune when related and out of tune when unrelated. The proportion of targets judged to be in tune was significantly greater than chance when related $(.56), t(14)=2.75, p<.01$, and significantly less than chance when unrelated $(.38), t(14)=4.66, p<.001$, where chance was .5 .

Thirteen out of the 15 subjects showed the reaction time advantage for related in-tune targets over unrelated in-tune targets. The size of this effect for the 13 ranged from $53 \mathrm{~ms}$ to 474 ms. The other 2 subjects, with 1 and 9 years of training, showed differences of $49 \mathrm{~ms}$ and $6 \mathrm{~ms}$, respectively. Reaction time differences were not correlated significantly with musical train$\operatorname{ing}(r=.301)$.

True identification times, corrected for guessing, showed similar patterns.

\section{Discussion}

The results of this experiment demonstrate priming of minor chords. This supports the hypothesis that the apparent absence of priming for minor targets in Experiment 1 was due to the greater stability or consonance of the alternative (major) target.

\section{General Discussion}

In this article we explored the use of reaction time to investigate the cognitive processes underlying harmonic expectation. A harmonic context primes the processing of chords related to the context, relative to chords unrelated to the context. In a speeded decision task, this shows up as an overall increased sensitivity (faster and more accurate responses) to related targets relative to unrelated targets, and a bias in favor of judging a related target to be the more stable or consonant of the two tar- 
get alternatives. This bias enhances the observed facilitation of the more stable or consonant target but diminishes it for the more unstable or dissonant one. Thus, minor chords seemed not to show evidence of priming in a major/minor decision task, but did show priming in an in-tune/out-of-tune task.

Marin and Barnes (1985) found that when subjects were asked to judge the relatedness of two chords, amateur musicians were faster at identifying them as related than as unrelated, but professional musicians and subjects with no musical training showed no difference. In Experiments 2 and 3 of our article, for which even untrained subjects were able to reach the criterion of accuracy, we found no significant correlation between priming and musical training. This suggests that a decision task such as in-tune/out-of-tune can fruitfully tap the underlying processes of listeners of all levels of training.

\section{References}

Bharucha, J. J. (1984). Anchoring effects in music: The resolution of dissonance. Cognitive Psychology, 16, 485-518.

Bharucha, J., \& Krumhansl, C. L. (1983). The representation of harmonic structure in music: Hierarchies of stability as a function of context. Cognition. 13, 63-102.

Bharucha, J. J., Olney, K. L., \& Schnurr, P. P. (1985). Coherence-disrupting and coherence-conferring disruptions in text. Memory \& Cognition, 13, 573-578.

Bharucha, J. J., \& Pryor, J. H. (in press). Disrupting the isochrony underlying rhythm: An asymmetry in discrimination. Perception \& Psychophysics.

Deutsch, D. (1969). Music recognition. Psychological Review, 76, 300307.

Dowling, W. J., \& Harwood, D. L. (1985). Music cognition. New York: Academic Press.

Jones, M. R. (1981). Music as a stimulus for psychological motion: Part I. Some determinants of expectancies. Psychomusicology, 1, 34-51.

Jones, M. R. (1982). Music as a stimulus for psychological motion: Part II. An expectancy model. Psychomusicology, 2, 1-13.

Krumhansl, C. L., Bharucha, J., \& Castellano, M. A. (1982). Key dis- tance effects on perceived harmonic structure in music. Perception \& Psychophysics, 32, 96-108.

Krumhansl, C. L., Bharucha, J. J., \& Kessler, E. J. (1982). Perceived harmonic structure of chords in three related musical keys. Journal of Experimental Psychology: Human Perception and Performance, 8. 24-36.

Krumhansl, C. L., \& Kessler, E. J. (1982). Tracing the dynamic changes in perceived tonal organization in a spatial representation of musical keys. Psychological Review, 89, 334-368.

Mandler, G. (1984). Mind and body: Psychology of emotion and stress. New York: Norton.

Marin, O. S. M., \& Barnes, S. (1985, August). Evidence for higher cognitive structures in music perception. Paper presented at the Fifth Workshop on Physical and Neuropsychological Foundations of Music, Ossiach, Austria.

Meyer, L. (1956). Emotion and meaning in music. Chicago: University of Chicago Press.

Piston, W. (1978). Harmony (4th ed.). New York: Norton.

Roberts, L. A. (1983, May). Consonance and dissonance judgments of musical chords. Paper presented at the 105 th meeting of the Acoustical Society of America.

Roberts, L. A., \& Shaw, M. L. (1984). Perceived structure of triads. Music Perception, 2, 95-124.

Rosner, B. S., \& Meyer, L. B. (1982). Melodic processes and the perception of music. In D. Deutsch (Ed.), The psychology of music. New York: Academic Press.

Schenker, H. (1954). Harmony (O. Jones, Ed., E. M. Borgese, Trans.). Cambridge: MIT Press. (Original work published 1906)

Schenker, H. (1979). Free composition (E. Oster, Ed. and Trans.). New York: Longman. (Original work published 1935)

Schoenberg, A. (1969). Structural functions of harmony (L. Stein, Ed.). New York: Norton. (Original work published 1954)

Shepard, R. N. (1964). Circularity in judgments of relative pitch. Journal of the Acoustical Society of America, 36, 2346-2353.

Wolford, G. L., Wessel, D. L., \& Estes, W. K. (1968). Further evidence concerning scanning and sampling assumptions of visual detection models. Perception \& Psychophysics, 3, 439-444.

Received December 20, 1985

Revision received May 16, 1986

\section{Change in Distribution of APA Convention "Call for Programs"}

In an effort to facilitate distribution of the APA "Call for Programs" for the annual convention, the "Call" for the 1987 convention will appear in the December issue of the APA Monitor instead of being a separate mailing to APA members. The 1987 convention will be in New York from August 28 to September 1. Deadline for submission of program and presentation proposals is January 20, 1987. Additional copies of the "Call" will be available from the APA Convention Office in December. 